\title{
Application of Surfactant Material as Anti-hygroscopicity Agent for Ammonium Nitrate $\left(\mathrm{NH}_{4} \mathrm{NO}_{3}\right)$
}

\author{
Baha Eldien Ismail Abd Allah Elzaki *§ \\ ${ }^{*}$ Chemcial Engineering Department, Karary University, Khartoum, 12304, Sudan \\ §bahaelzaki@karary.edu.sd
}

\begin{abstract}
Ammonium nitrate can be used as oxidizer in missile propellant. It is present as the main element in many manufacturing explosives. Due to the surface polarity of ammonium nitrate, the particles can easy absorb moisture. In this study, ammonium nitrate particles were coated by cetylalcohol surfactant due to decrease the hygroscopicity. The optimized physical coating process using cetylalcohol was achieved by $\left(L^{9}\left(3^{4}\right)\right)$ Taguchi orthogonal array (TOA).The analysis of TOA revealed that the highest decline of absorption rate was $35.45 \%$ with the mass ratio of coating layer was $0.95 \%$. Scanning electron microscopy (SEM) was used to characterize the surface of coated and uncoated ammonium nitrate. The idea and approach presented in this study can support the researchers to improving anti-hygroscopicity of ammonium nitrate.
\end{abstract}

Key words-Ammonium nitrate (AN), Cetylalcohol, Hygroscopicity, Physical coating, Surfactant material

\section{INTRODUCTION}

Ammonium nitrate (AN) has many uses in a fertilizer and energetic materials, such as explosives and propellants [1]. AN particles is obtainable, inexpensive value, and easy to manufacture [2]. In comparison, ammonium perchlorate (AP) have a significant impairment to the surroundings area when used as oxidizer, thus, AN particles will come to be a potential replacement after handling its disadvantage such as hygroscopicity, phase transitions, and low burning rate [2][4]. Conversely, the AN particles it can easy absorb moisture from surrounding environmental [2], [4]-[6]. The high polarity surface of AN growths the possible of the molecules to absorb moisture [7]. Many research has been done on surface modification of AN particles to reduce the hygroscopicity, such as using a physical coating by $\mathrm{C}_{n} \mathrm{H}_{2 n+2}, \mathrm{C}_{18} \mathrm{H}_{36} \mathrm{O}_{2}$, mineral oil coated on the surface of the ammonium nitrate particles, making a hydrophobic coating. Physical coating method is simple, suitable, easy, and safety in industrialized [8].

Furthermore, the chemical coating method was employed to decrease absorb moisture of AN by surfactants, coupling agents and other efficient groups in organic molecules. While chemical coating method used a smaller amount quantity and strong binding force, the molecular weight of coating materials is quite minor, low hydrophobic properties [8]-[10].

Moreover, in 2013, Zhicheng et al. used in conditions of a temperature of $35 C^{\circ}$, a relative humidity of $92 \%$, and an absorption moisture time of $24 \mathrm{~h}$ and chloroform as a solvent, and the reaction time was $8 \mathrm{~h}$ at $65 C^{\circ}$ to reduce hygroscopicity by methyl methacrylate as a monomer in precipitation polymerization, and azobisisobutyeonitrile as an initiator. Under the absorption rate of the moisture was reduced from $12.26 \%$ to $8.78 \%$, the decline was $28.35 \%$, and the mass ratio of the coating layer was 0.48 [11], [12]. From the pervious, the literature of reducing moisture of AN particles using a different coating materials and coating technique, obtained under confident conditions, absorption moisture rate a large decline-coated sample, although significantly modified antihygroscopicity AN particles [13].

Conversely, many tasks are residual. For instance, the index evaluation of the AN particles hygroscopic effect did not precisely mention the mass ratio of the coating layer. Moreover, the hygroscopicity test conditions of AN particles coated keep on undistinguishable. As example, the time of moisture absorption is fairly small, although both the particle size of AN and the size of weighting bottle are unidentified, as well as the process of coated AN particles by surfactant agent have not been systematically declared, mainly the coating time and temperature. In this study, the improved physical coating process using cetylalcohol was completed by $\left(L^{9}\left(3^{4}\right)\right)$ Taguchi orthogonal array (TOA) to reduce the hygroscopicity. TOA applied to explore convinced factors affecting the coating process before optimizing our formula in terms of fixed reaction temperature, time of fixed reaction temperature, the amount of surfactant, and time of reducing temperature. Furthermore, the surface morphologies of AN particles were characterized by electronic scanning microscope (SEM).

\section{MATERIALS AND METHOD}

\section{A. Materials}

Ammonium nitrate supplied by Kecheng fine chemical CO. Ltd ( $\geq 99.0 \%$ Shanghai, China), chloroform ( $\geq \%$, Shanghai Ling Feng Chemical Reagent Co. LTD, China), cyclohexane and strontium chloride hexahydrate $(\geq 99.0 \%$ Chengdu Kelong Chemical Reagent, China).cetylalcohol $(\geq 0.99 \%$ Chengdu Kelong Chemical Reagent, China), deionized water 


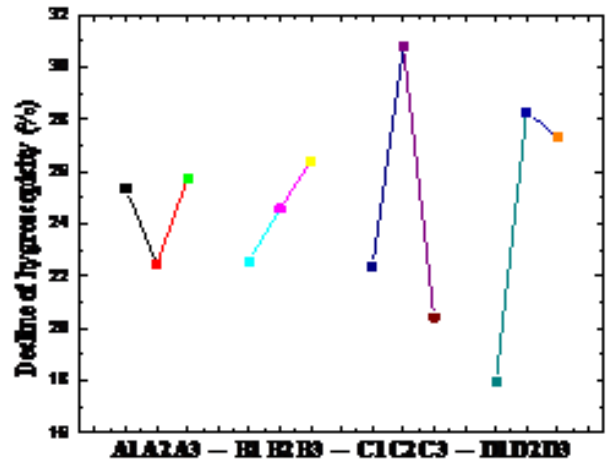

Fig. 1. Orthogonal experiment of coating AN by cetylalcohol

(Nanjing University of science and technology water center).

\section{B. Method}

Ammonium nitrate was coated by physical coating methods to reduce hygroscopicity [7], [14]. $6 \mathrm{~g}$ of AN particles coated by cetylalcohol in the blend of solvents contain of $15 \mathrm{ml}$ chloroform and $15 \mathrm{ml}$ cyclohexane, selecting the solvents for coating based on the solubility of surfactant agent, and AN particles is not soluble in this solvents. The coating process conducted at constant temperature $60 C^{\circ}$. After $2 \mathrm{~h}$ the heating was stopped, and then the temperature was slowly decreased within $8 \mathrm{~h}$ to $30 C^{\circ}$, at this stage the water of bath heating was replaced by cool water, and when the temperature reached to $25 C^{\circ}$. AN coated was filtered by vacuum filter, and dried at a certain temperature with considering the melting and boiling point of each surfactant materials.

\section{Moisture absorption rate}

In order to estimate the performance of coating by surfactant materials it is necessary to know the moisture absorption rate at a constant temperature and relative humidity conditions. The moisture absorption rate of coated and uncoated AN particles was determined according to Chinese standards GJB770B - 2005 "hygroscopic - drier balance method", after modified the conditions test in our group to $5 \mathrm{~g}$ AN in $6030 \mathrm{~mm}$ weighing bottle at temperature of $35 \mathrm{C}^{\circ}$, humidity $(67.5 \%)$, and time of absorption rate tested $24 \mathrm{~h}$. The moisture absorption rate (HR) was calculated by the following equation:

$$
H R=\frac{M-M_{0}}{M_{0}} \times 100 \%
$$

Where: $H R$ - moisture absorption rate, $\%$.

$M_{0}$ - The weight of sample before absorbed moisture, g. $M$ - The weight of the sample after absorbed moisture, $g$

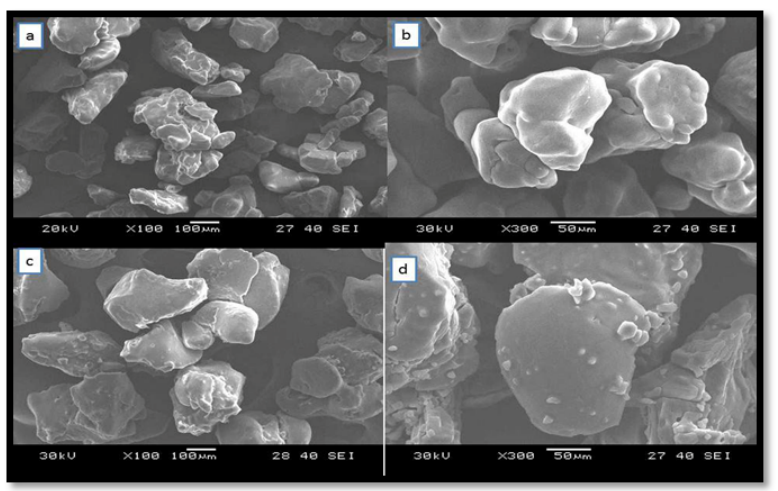

Fig. 2. Morphology of AN/cetylalcohol (a, b), and uncoated AN (c, d)

TABLE I

ATTRIBUTION OF LEVELS TO FACTORS IN $L^{9}\left(3^{4}\right)$ TOA EXPERIMENTS

\begin{tabular}{llll}
\hline Factors & Levels \\
& 1 & 2 & 3 \\
\hline A: Fixed reaction temperature & 55 & 60 & 65 \\
B: Time of fixed reaction temperature & 1.5 & 2 & 2.5 \\
C: Amount of Surfactant & 1 & 1.5 & 2 \\
D: Time of reducing temperature & 7 & 8 & 9 \\
\hline
\end{tabular}

TABLE II

$L^{9}\left(3^{4}\right)$ TOA WITH EXPERIMENTAL RESULTS OF AN/CETYLALCOHOL

\begin{tabular}{llllllll}
\hline No. & A & B & C & D & $\begin{array}{l}\text { HR } \\
(\%)\end{array}$ & $\begin{array}{l}\text { W } \\
(\%)\end{array}$ & $\begin{array}{l}\text { A } \\
(\%)\end{array}$ \\
\hline 1 & 1 & 1 & 1 & 1 & 10.92 & 0.82 & 14.69 \\
2 & 1 & 2 & 2 & 2 & 7.81 & 0.95 & 35.45 \\
3 & 1 & 3 & 3 & 3 & 8.96 & 1.27 & 25.95 \\
4 & 2 & 1 & 2 & 3 & 8.52 & 1.50 & 29.59 \\
5 & 2 & 2 & 3 & 1 & 10.67 & 1.41 & 11.82 \\
6 & 2 & 3 & 1 & 2 & 8.96 & 1.13 & 25.95 \\
7 & 3 & 1 & 3 & 2 & 9.26 & 0.55 & 23.47 \\
8 & 3 & 2 & 1 & 3 & 8.90 & 1.22 & 26.45 \\
9 & 3 & 3 & 2 & 1 & 8.77 & 0.87 & 27.34 \\
k1 & 25.36 & 22.58 & 22.36 & 17.95 & & & \\
k2 & 22.45 & 24.57 & 30.79 & 28.29 & & & \\
k3 & 25.75 & 26.41 & 20.41 & 27.33 & & & \\
R & 02.91 & 03.83 & 10.38 & 10.34 & & & \\
\hline
\end{tabular}

TABLE III

VERIFICATION EXPERIMENT OF THEORETICAL OPTIMUM AND THE ACTUAL BEST RESULTS OF CETYLALCOHOL

\begin{tabular}{lllllll}
\hline & HR & W & & Blank AN & A & Average (A) \\
No. & & & Average (W) $(\%)$ & & & \\
& $(\%)$ & $(\%)$ & & $1 \%)$ & $(\%)$ \\
\hline 1 & 9.03 & 0.91 & & 12.10 & 25.37 & \\
2 & 8.07 & 0.77 & 0.85 & 10.74 & 24.86 & 24.44 \\
3 & 8.26 & 0.86 & & 10.74 & 23.09 & \\
1 & 7.81 & 1.32 & & 12.10 & 35.45 & \\
2 & 7.31 & 1.15 & 1.26 & 10.74 & 31.93 & 32.51 \\
3 & 7.50 & 1.32 & & 10.74 & 30.17 & \\
\hline
\end{tabular}




\section{Mass ratio of coating layer test}

The method determined the mass ratio of coating layer was developed to reach additional accurateness as following steps: Firstly: The diameter of filter paper was $18 \mathrm{~cm}$ wetted by using deionized water.

Secondly: An oven used at temperature $100 \mathrm{oC}$ to dry the filter paper the time of trying $1 \mathrm{~h}$, then the weight of filter paper was measured (WB).

Thirdly: The coated AN particles after absorption moisture was dissolved in deionized water, and filtered through a tapered funnel, and the filter paper was washed several times by deionized water, and placed in an oven at $100 \mathrm{oC}$ for $1 \mathrm{~h}$. Finally, the filter paper was measured again $\left(W_{A}\right)$.

The mass fraction of coating layer was calculated by following equation:

$$
W=\frac{M_{0}}{M_{1}} \times 100 \%
$$

Where: W- mass ratio of coating layer, $\% . M_{0}$ - The weight of sample before absorbed moisture, g. $M_{1^{-}}$The weight of coating layer, $g=W_{A}-W_{B}$.

\section{E. Decline of moisture absorption rate}

The decline of moisture absorption rate was calculated by following equation:

$$
A=\frac{H R_{1}-H R_{2}}{H R_{1}} \times 100
$$

Where: $A$ - decline of moisture absorption rate, \%. $H R_{1}$ absorption rate of AN sample, \%. $H R_{2^{-}}$absorption rate of coated AN sample, $\%$.

\section{F. Taguchi orthogonal array (TOA)}

A $L^{9}\left(3^{4}\right)$ TOA was used in the current study to define the optimal conditions regarding the selected factors which affect the coating process of AN with cetylalcohol. The design involved four factors at three levels as shown in Table (I).

These factors were a fixed reaction temperature, time of fixed reaction temperature, amount of surfactant, and time of reducing temperature. As shown in Table III, the $L^{9}\left(3^{4}\right)$ array had 9 rows and four columns at three levels.

Table I. Attribution of levels to factors in $L^{9}\left(3^{4}\right)$ TOA experiments

\section{G. Characterization}

The surface modified of ammonium nitrate particles were observed for their morphological characterization study under scanning electron microscopy (JEOL JSM 6380LV, Japan), and used AN without coated as a control.

\section{RESULTS AND DISCUSSION}

\section{A. Taguchi orthogonal array (TOA)}

The results of orthogonal optimization experiments of AN particles coated by $C_{16}$-alcohol, as shown in Table II, the $L^{9}$ $\left(3^{4}\right)$ array had 9 experiments and four factors at three levels as showed in Table I.

Table II. $L^{9}\left(3^{4}\right)$ TOA with experimental results of AN/cetylalcohol

Table II and Figure 1 showed that the $\mathrm{R}$ of processing factors in the orthogonal experiment ranged its values in order, $R_{C}>R_{D}>R_{B}>R_{A}$. This process had four factors for their $R$ to be from strong to weak affecting on the anti-hygroscopicity performance of AN particles coated with cetyalcohol.

These factors were the temperature of coating process, the time of coating process, the amount of surfactant, and the time of decreasing temperature. As showed in Figure 1, the conditions of theoretical optimum coating process of AN particles coated with $C_{16}$-alcohol to reduce hygroscopicity, namely $\mathrm{A} 3 \mathrm{~B} 3 \mathrm{C} 2 \mathrm{D} 2$ were: the temperature of coating process was $65 \mathrm{C}^{\circ}$, the time of coating process was $2.5 \mathrm{~h}$, the amount of surfactant was $1.5 \mathrm{~g}$, and the time of decreasing temperature was $8 \mathrm{~h}$. The conditions of actual optimum coating process, namely $\mathrm{A} 2 \mathrm{~B} 2 \mathrm{C} 2 \mathrm{D} 2$ were that, the temperature of coating process was $60 C^{\circ}$, the time coating process was $2 \mathrm{~h}$, the amount of surfactant was $1.5 \mathrm{~g}$, and the time of decreasing temperature was $8 \mathrm{~h}$. Furthermore, optimum processes in both theoretical and actual level were repeated for verifying, the experimental results were shown in Table III.

It could be seen from Table 3 that the repeated average decline of hygroscopicity of the coated samples of theoretical optimum results of the experiments was $24.44 \%$, with an repeated average mass ratio of the coating layer was $0.85 \%$, while the repeated average decline of hygroscopicity of the actual optimum point of the experiments was $32.51 \%$, the repeated average mass ratio of the coating layer was $1.26 \%$. The antihygroscopicity performance of actual optimum results was better than that of theoretical best points. Therefore, the optimum coating conditions of the anti-hygroscopic surface modification of AN particle coated by Cetylalcohol were as follows: the temperature of coating process was $55 \mathrm{C}$, the time of coating process was $2 \mathrm{~h}$, the amount of surfactant was $1.5 \mathrm{~g}$, and the time of reducing temperature was $8 \mathrm{~h}$.

The optimum results were as follows: the decline of hygroscopicity was $32.51 \%$ and the mass ratio of coating layer was $1.26 \%$ under the moisture absorption test conditions, the temperature was $35 \mathrm{C}$, the relative humidity was $68 \%$ and the time of hygroscopicity test was $24 \mathrm{~h}$.

\section{B. Scanning Electron Microscopy (SEM)}

Different scale bar of $100 \mathrm{~m}$, and $50 \mathrm{~m}$ Electron micrographs demonstrated in figure 2 respectively. This figure, showed that the characterization of the surface morphology of the AN particle coated by cetylalcohol. The Sample No.2 in Table II $(a, b)$ which showed a difference in the shape particles and the surface smooth without cracking in surface coating layer as compared to AN particle without coating (c, d). Moreover, the difference between the coated AN particle and AN particle without coating significance displayed.

\section{CONCLUSION}

AN particles coated by cetylalcohol to reduce hygroscopicity. The optimized modification process of coating AN particles 
were successfully prepared as a result of TOA analysis. The highest decline of absorption rate was $35.45 \%$ with the mass ratio of coating layer was $0.95 \%$. The cetylalcohol showed a promising coating surfactant agent for AN particle, and it should be exposed for advance investigations as antihygroscopicity.

\section{REFERENCES}

[1] Nagayama, S., et al., Differential scanning calorimetry analysis of crystal structure transformation in spray-dried particles consisting of ammonium nitrate, potassium nitrate, and a polymer [J]. Journal of Thermal Analysis and Calorimetry, 2014. 118(2): 1215-1219.

[2] Rolf, F.-M., Production of ammonium nitrate [P]. 1972, U.S. Patent $3,690,820$.

[3] Oommen, C. and S. Jain, Ammonium nitrate: a promising rocket propellant oxidizer [J]. Journal of hazardous materials, 1999. 67(3): 253-281.

[4] Chaturvedi, S. and P.N. Dave, Review on thermal decomposition of ammonium nitrate [J]. Journal of Energetic Materials, 2013. 31(1): 126.

[5] Nagayama, S., et al., Moisture Proofing of Spray Dried Particles Comprising Ammonium Nitrate/Potassium Nitrate/Polymer [J]. Propellants, Explosives, Pyrotechnics, 2015. 40(4): 544-550.

[6] Elzaki B I, Zhang Y J. Anti-hygroscopic surface modification of ammonium nitrate (NH4NO3) coated by surfactants [J]. Arabian Journal of Chemistry, 2018, 13(1):3460-3473.

[7] Wei, Y. and B.H. Cai. Study on Surface Modification of Ammonium Nitrate [J]. Advanced Materials Research. 2012. Trans Tech Publ.

[8] Elzaki, B.I. and Y.J. Zhang, Surface modification of ammonium nitrate by coating with surfactant materials to reduce hygroscopicity [J]. Defence Technology, 2019. 15(4): 615-620.

[9] Malash, G. and H. Hashem, Improving the properties of ammonium nitrate fertilizer using additives [J]. Alexandria Engineering Journal, 2005. 44(4): 685-693.

[10] Zhang, X.-d., et al., Surface Modification of Phase Stabilized Ammonium Nitrate and Its Application in Solid Composite Propellants [J]. Chinese Journal of Explosives \& Propellants, 2009. 1: .02.

[11] Zhang, J. and R.-j. Yang, Study on Surface Properties of Coated Ammonium Nitrate [J]. Energetic materials-chengdu-, 2004. 12(1): 1-5.

[12] Elzaki, B.I. and Z.Y. Jun, Relationships between structures of surfactants and their anti-hygroscopicity performance of ammonium nitrate particles [J]. Arabian Journal of Chemistry, 2020. 13(11): 7626-7636.

[13] Elzaki, B.I. and Y.J. Zhang, Coating Methods for Surface Modification of Ammonium Nitrate: A Mini-Review [J]. Materials, 2016. 9(7): 502 -512 .

[14] Xiong, X. and Z. Liu, Improvement of the Hygroscopicity of AN by Modified Paraffin [J]. Chin. J. Explos. Propell, 2013. 36:. 50-52. 